\title{
INVESTIGACIÓN/RESEARCH
}

Recibido: 10/11/2012-----Revisado: 22/01/2013 Aceptado: 30/04/2013-----Publicado: 15/07/2013

\section{ENCRUCIJADA DOCENTE: LA INCLUSIÓN DE LAS TECNOLOGÍAS DE LA INFORMACIÓN Y COMUNICACIÓN}

Javier Rodríguez Torres ${ }^{1}$ : Universidad de Castilla-La Mancha. España.

javier.rtorres@uclm.es

\section{RESUMEN}

El artículo surge como producto de la indagación y asesoramiento en distintos centros de Educación Primaria y Secundaria de la provincia de Toledo. El objeto de estudio de nuestro interés, fue conocer cómo los docentes abordaban su práctica cotidiana y los sus propios procesos de formación, formales o no, ya iniciados, en relación a la inclusión de las Tecnologías de la Información y la Comunicación. Extrajimos lo más relente de sus discursos, contrastando con otros ya existentes en la literatura pedagógica, referentes al objeto de estudio y adyacentes, para extraer conclusiones que nos hagan seguir avanzando o retomando procesos poco consolidados.

PALABRAS CLAVE: Formación docente - Currículum - Cultura tecnológica Interdisciplinariedad.

\footnotetext{
${ }^{1}$ Autor correspondiente:

Javier Rodríguez Torres: Profesor de la Facultad de Educación de Toledo de la Universidad de Castilla - La Mancha. España.

Correo: iavier.rtorres@uclm.es
} 


\title{
EDUCATIONAL CROSSROADS: THE INCORPORATION OF THE TECHNOLOGIES OF THE INFORMATION AND COMMUNICATION
}

\begin{abstract}
The article arises as product of the investigation and advice in different centers of Primary and Secondary Education of the province of Toledo. The object of study of our interest, it was to know how the teachers were approaching his daily practice and his own processes of formation, formal or not, already initiated, in relation to the incorporation of the Technologies of the Information and the Communication. We extracted more dampness of his speeches, contrasting with already existing others in the pedagogic literature, modals in order to study and adjacent, to extract conclusions that make us continue advancing or taking again little consolidated processes.
\end{abstract}

KEYWORDS: Educational formation - Curriculum - Technological culture Interdisciplinary.

Los alumnos van por delante de nosotros, el desfase existe...

Llegas a tu clase pensando que les Ilevas algo muy novedoso...

Ilegas al chico: imira hay esto! Ves que te mira y parece decirte:

"iyo voy, ya, dos pueblos por delante!". Los chavales van bastante

más deprisa que nosotros... bastante más.

(Entrevista profesor de IES)

\section{INTRODUCCIÓN}

Aunque resulte reiterativo, el docente es un elemento esencial en la calidad de la educación, sin ser el único, todos los demás elementos cobran sentido y pasan por él. Sin temor a equivocarnos, en los sistemas educativos, el docente es el elemento mediador de otros que no depende de él: familias, recursos, estructuras organizativas².

Desde nuestras investigación y asesoramiento a centros, una formación permanente que se centra en destrezas concretas sin tener en cuenta al $<<$ docente total $>>$, queremos decir, con sus propias características personales de edad, género, experiencias y valores personales construidos de forma permanente en contextos sociales, está contribuyendo a acentuar lo inadecuado del planteamiento y el problema en general, en nuestro caso la inclusión de las Tecnologías de la Información y Comunicación (en adelante TIC)

\footnotetext{
${ }^{2}$ GIMENO (1996: 15) planteaba que "no deja de ser sospechoso (que) vuelva a ponerse el énfasis en las estructuras organizativas sin darse cuenta de que el profesorado es el elemento capital de cualquier reforma educativa y hay que animarlo, estimularlo, pagarle y exigirle, por ese orden."
} 
Así, desde este artículo queremos profundizar en las vicisitudes en las que está inmerso el docente: qué pasa, qué hace con las TIC en su entorno cotidiano - el aula - y, por otra parte, cómo se siente, cómo se sitúa ante la formación en estos y con estos contenidos.

\section{METODOLOGÍA}

La investigación presentada pretende conjugar, desde una perspectiva naturalista, el conocimiento que proporcionan de una parte, la experiencia profesional de los docentes en el ámbito de la actualización profesional realizada en materia de TIC, la interpretación y la reflexión sobre ello, y de otra parte, los esquemas pedagógicos sobre la integración de las TIC en la escuela que se definen y desarrollan en las actividades de formación.

La perspectiva de investigación por la que optamos es coherente con la definición del objeto de estudio. Planteamos comprender cómo los docentes se acomodan a los fines y contenidos de su actualización profesional sobre la integración escolar de las TIC, y analizar qué valor otorgan a esta experiencia en relación con su trabajo en los centros. Esta realidad conduce a un planteamiento epistemológico que asume una visión dinámica y subjetiva de la práctica educativa: el componente motivacional y compromisos de los docentes, -claves para comprender las acciones que emprenden-, cuyo conocimiento permitirá definir pautas y contenidos para su formación y actualización profesional.

El tipo de diseño por el que optamos es de corte etnográfico. Como explican Goetz y LeCompte, (1988), estos diseños intentan describir y reconstruir de forma sistemática características de las variables y fenómenos, con el fin de generar y perfeccionar categorías conceptuales, descubrir y validar asociaciones entre fenómenos, 0 comparar constructor y postulados generados a partir de fenómenos observados en escenarios distintos.

Hemos utilizado, por tanto, desde nuestras necesidades de reflexión sobre la práctica, una metodología de investigación de tipo cualitativo de corte etnógrafo. Con instrumentos de recogida de datos: los cuestionarios, la observación directa, las entrevistas y reconstrucción de biografías docentes en relación con su perfeccionamiento y actividades de desarrollo personal, y los grupos de discusión.

\section{ANÁLISIS Y DISCUSIÓN}

\subsection{En su cotidianidad.}

Resultan significativos son los datos que hemos recogido durante nuestro trabajo en los centros:

- los alumnos tienen el poder estratégico porque dominan el medio, buscan información, se comunican, <<bajan>> música, son en definitiva consumidores de la cultura popular.

- los estudiantes exhiben un comportamiento autónomo ante las experiencias y mediaciones que experimentan en los contextos externos que no ajenos a la 
escuela, lo que les lleva incluso a cuestionar las metodologías y estrategias de aprendizaje tradicionales que la escuela sigue manteniendo como únicas y válidas.

- realizan otros aprendizajes que la escuela debiera perfeccionar, como es la búsqueda de información que bajo estrategias de ensayo-error les lleva a los alumnos a ser conscientes de la necesidad de que en el espacio escolar les permitieran perfeccionar las estrategias de acceso a la información.

- se ha evidenciado el consumo acrítico de contenidos asociados a la industria cultural, pero que son contenidos que les interesan y les mueven a experimentar de un modo autónomo con las herramientas tecnológicas.

Estamos evidenciando que una parte significativa de profesores en los centros escolares permanece pasivo, que no ajeno, a las formas y contenidos de apropiación informal de la tecnología que realiza una parte significativa de los alumnos. Comienzan a evidenciar que sus alumnos se familiarizan con el ordenador a través de los grupos de iguales o solos en sus hogares, cuyo contexto familiar tiene un grado de aceptación significativo de la cultura tecnológica. Algunos profesores reconocen su práctica educativa como algo ajeno a toda innovación tecnológica, siendo conscientes que las experiencias vitales de sus alumnos comienzan a suponer un $<<$ currículum paralelo >> (Gimeno Sacristán, 1999) al curriculum establecido. E Incluso el profesorado que lleva a cabo usos poco significativos de las TIC es consciente de la necesidad de seguir avanzando y enriqueciendo los usos que llevan a cabo. Entran de este modo a tomar conciencia que la escuela ha dejado de tener el $<<$ monopolio del saber >> (Carbonell, 2001), y sienten que parte del conocimiento escolar se devalúa.

Ante estas circunstancias una gran parte del profesorado suele mantener dos posturas mayoritarias, que forman parte de sus creencias profesionales y se convierten en un obstáculo para una inclusión de las TIC en el currículo de un modo regular y como sustentadora de una utilización didáctica globalizadora y transversal.

\section{Postura Primera}

Podríamos denominar perspectiva determinista, se adapta a los desarrollos tecnológicos y sus aplicaciones sin un análisis en profundidad de la <<utilidad>> educativa de estos medios desde referentes curriculares y pedagógicos de más largo alcance. La cultura tecnológica se reduce a un conocimiento de tipo instrumental, de destrezas técnicas, al servicio de la enseñanza. La utilización de los medios electrónicos para presentar contenidos conceptuales, organizarlos y motivar a los estudiantes son las razones que se esgrimen.

Otras razones que suelen aducirse para justificar este tipo de inclusión es simplemente que si la tecnología está presente en $<<$ nuestras vidas >> la escuela no puede dar la espalda a esta evidencia, los docentes suelen entender además que la familiarización y dominio instrumental de estos recursos por parte de los estudiantes le facilitará en cierto modo una salida en el mercado laboral.

En sus concreciones prácticas se reproducen los planteamientos dominantes en la formación del profesorado basados en el expertismo. Desde esta postura la inclusión 
de estos recursos pone énfasis en las nociones, conceptos y datos necesarios para utilizar estos recursos a nivel de usuario por parte del profesorado, bien para una utilización disciplinar o bien especializada como corresponde a las clases de informática. En sí misma, no es una perspectiva negativa, incluso podemos entenderla como una fase necesaria, el peligro que encierra es que puede consolidarse como única alternativa posible y válida.

Tras esta postura se plantea la inclusión curricular de las TIC de un modo asertivo: "tecnologías $s f^{\prime}$, pero desde lo que puede hacer la aplicación de turno. Esta postura se concreta en prácticas escolares que desestiman otras metas acordes con la sociedad de la información y otros enfoques de enseñar y aprender. Hemos constatado la dificultad que el profesorado tiene para identificar otros aprendizajes y otras prácticas educativas más relevantes pedagógicamente. $\mathrm{Y}$ lo que también es importante, se obvia comprender el modelo de enseñanza y aprendizaje lineales y reproductores que subyacen en las acciones prácticas en que se concretan estos planteamientos.

\section{Postura Segunda}

Existe otra postura, contradictoria $y$, en cierto modo, ambivalente: "hay que incorporar la TIC en la escuela, no hay duda, pero....". Algunos docentes saben de las experiencias que sus alumnos vivencian con estos recursos, bien desde su rol de padres y madres, bien desde su rol como profesionales situados en las coordenadas socioculturales actuales. Pero el qué incorporar de la cultura tecnológica y cómo llevarlo a cabo es para el profesorado en estos momentos una deliberación pendiente, no exenta de obstáculos e incertidumbres.

Buscar respuestas no es fácil para ellos, porque esta postura suele contraponer cultura impresa a cultura tecnológica, una enseñanza basada en medios impresos frente a una enseñanza basada en medios electrónicos. De este modo se corre el riesgo de que los medios electrónicos salgan perdiendo en la comparación, pudiéndose concluir que los medios impresos han cumplido siempre mejor con los fines de la educación obligatoria. Esta postura no desestima que las tecnologías puedan aportar algo a la educación de los estudiantes, pero esta aportación no suele percibirse como algo relevante a su tarea de enseñar si atendemos a como a la postre se concretan las prácticas escolares. Incluso se puede llegar a conclusiones más radicales: que la cultura tecnológica es un conjunto de prácticas que atenta contra la cultura escolar. Y mientras los <<porqué>> no estén claros, los videojuegos, la Internet, los SMS... y los usos a ellos asociados pueden llegar a ser vistos como una realidad negativa, incluso amenazante, que dificulta la tarea docente.

Con la mirada puesta en las prácticas dominantes que prevalecen en los centros escolares, y desde su experiencia profesional -hagan o no uso de estos medios-, algunos docentes van asumiendo que las clases de informática o el uso puntual del ordenador no es una práctica sustentadora de los importantes aprendizajes que sus alumnos tienen que llevar a cabo. Al oponer enseñanza basada en medios tradicionales frente a una enseñanza basada en medios electrónicos, se apela de un modo implícito a una idealización de la enseñanza y del aprendizaje basado en 
modelos tradicionales, impidiéndose un análisis crítico sobre el alcance de la enseñanza basada en los medios de siempre. Cuestión importante si tenemos en cuenta las consecuencias que para la educación obligatoria tiene la $<<$ política del libro de texto >> (Martínez Bonafé, 2002). De este modo, algunos docentes aunque en su discurso no rechazan abiertamente la incorporación y uso de las tecnologías, en la práctica acaban adoptando una actitud de oposición pasiva ante su integración curricular.

Nuestra intención en este trabajo, no es exponer sólo lo $<<$ negativo $>>$, aunque constatado durante nuestra indagación; queremos recalcar otros casos en que nos encontramos con acciones docentes, que aunque minoritarias, son claras y conscientemente posiciones profesionales ante la inclusión de las TIC en la escuela superadores del $<<$ determinismo tecnológico $>>$. Algunos profesores están siendo protagonistas del diseño y la puesta en práctica de verdaderos procesos de cambio en la inclusión curricular de las TIC, cuyos planteamientos necesitamos comprender.

En síntesis, desde las posturas mencionadas se están desestimando varias cuestiones que la formación del profesorado debiera de rescatar como contenido formativo. Entendemos que tras estos planteamientos los profesores no tienen en cuenta que las tecnologías y sus aplicaciones, por no ser independientes de otras prácticas sociales en las que sus alumnos se ven inmersos, tienen un alto contenido educativo que debe ser rescatado por la cultura escolar para sus perfeccionamiento desde principios educativos coherentes con la sociedad de la información (Pérez Gómez, 1998). Y a esta cuestión no le hemos prestado mucha atención hasta ahora, de modo que las necesidades sociales y educativas que la sociedad de la información plantea a la escuela no están claras.

Téngase en cuenta que la primera postura define contenidos y actividades desde un determinismo tecnológico. La segunda postura, si bien mira desde una perspectiva sociocultural a las TIC al reconocer los procesos de mediación y experiencia que vivencian los alumnos es los espacios externos a la escuela, no acaba de identificar esos procesos como fuente de conocimiento en clave pedagógica.

En definitiva, ambas posturas desestiman otros aprendizajes que en la actualidad son necesarios trabajar en la escuela, aprendizajes que serían la expresión más elaborada de las nuevas formas de comunicación, acceso a la información y nuevos contenidos que estos recursos nos brindan, y que en última instancia tienen que formar parte de los fines que persiga la escuela.

Es decir, el primer paso que debiéramos acometer sería poner al profesorado en situación de que comprendiera y dotara de sentido educativo a la integración curricular de las TIC desde una perspectiva de re-construcción social. Como plantean Fullan $(2002,103)$ cuando las razones que justifican un cambio no están claras, y el profesorado no ve la necesidad del cambio planteado, éste termina siendo más superficial y aparente, que real. El segundo paso, sería clarificar a qué fines educativos podría contribuir su utilización en la educación obligatoria. Téngase en cuenta, que para el profesorado integrar y hacer uso de las TIC desde lo que puede hacer los 
programas y aplicaciones, puede ser un objetivo claro, pero no lo suficientemente significativo como para que los docentes se pongan en situación de re-pensar y recrear sus prácticas educativas.

Por todo lo expuesto y observado hasta el momento entendemos que la formación del profesorado debiera de trabajar, como una primera fase o etapa, contenidos que permitan a los profesores desentrañar en clave pedagógica la relación interdisciplinar entre la cultura tecnológica y la cultura escolar. Las TIC en la formación del profesorado se convertirían en la posibilidad necesaria para que el docente:

1. Transformara en clave de aprendizajes los conocimientos que permiten a cualquier persona situarse en una sociedad interconectada, que caracterizada por flujos de información constantes y cambios socioculturales potentes ${ }^{3}$, requieren de destrezas, capacidades y actitudes propias de una educación transversal. ¿Por qué hay que integrar las tecnologías en los proyectos educativos? ¿Necesitan los alumnos saber algo nuevo? ¿Por qué? La búsqueda de razones y argumentos que justifiquen la utilización de las TIC más allá de los argumentos impuestos por la moda y el mercado, les facultaría para comprender las necesidades sociales y educativas que la sociedad de la información plantea a la escuela. Valga como ejemplo, la traducción educativa que nos hace Gimeno (2005) de los procesos y experiencias que las nuevas tecnologías de la información nos brindan, traducción que revitalizaría el proyecto de la educación obligatoria mediante nuevas y no tan nuevas experiencias de lectura y escritura y, por tanto, de acceso al conocimiento de la mano de estos medios.

2. Consecuentemente, también sería una oportunidad para que el docente analizara y clarificara qué grandes metas educativas podrían ser satisfechas y trabajadas en las aulas. Los profesores somos quienes traducimos y llenamos de significado los fines de la educación desde nuestras motivaciones, intereses y conocimiento profesional. Se trataría de subjetivizar lo $<<$ nuevo $>>$ en relación con las $<<$ viejas $>>$ metas, que sintieran que la utilización de las TIC es algo valioso que merece ser trabajado en las aulas porque $<<$ leen $>>$ con claridad los fines educativos. Esto no significa que la integración de las tecnologías tenga que ser traducida en pequeños fines, porque como nos recuerda Fullan los cambios simples e insignificantes pueden ser muy claros, pero poco relevantes en el momento de implementarlos y concretarlos en prácticas sólidas y de continuidad en los centros educativos.

Se trataría en definitiva que los docentes buscaran razones para la utilización de las tecnologías y sus aplicaciones en coherencia con las necesidades sociales y educativas que tiene planteadas la escuela. Una búsqueda debatida y consensuada para que las

\footnotetext{
${ }^{3}$ Piense el lector la necesaria reflexión que los profesores debemos de realizar sobre los cambios en los nuevos escenarios laborales, pues en su dinámica y consecuencias para los futuros trabajadores encontraríamos parte del sentido y la urgencia de facilitar a las nuevas generaciones procedimientos de trabajo intelectual para el aprendizaje autónomo.
} 
hicieran suyas desde sus compromisos e intereses. Todos los docentes tienen unas creencias más o menos explícitas sobre las razones y fines que satisface el uso de las tecnologías en la educación obligatoria, y son estos supuestos los que se tienen que someter a revisión. Entiendo que mientras estos contenidos no se rescaten en la formación del profesorado, y se anteponga su tratamiento a favor de la alfabetización meramente instrumental de los aparatos y aplicaciones, le estaremos ofreciendo pocas alternativas para una intervención de cambio positivo en la práctica educativa.

Una segunda fase o etapa se concretaría en poner a los docentes en situación de traducir lo fines identificados en la fase anterior en metodologías de trabajo. La integración y utilización de los medios electrónicos merece la pena ser planteada en torno a conflictos curriculares. El cómo llevar a cabo propuestas de trabajo no puede reducirse a cómo trabajar con los alumnos en función de las posibilidades del programa o aplicación del momento. El profesorado tiene que interpelarse y debatir qué pueden hacer en consonancia con los fines que hayan decidido son valiosos y merecen la pena trabajar en la fase anterior. Habría que convertir la formación en un espacio para que los docentes:

1. Desentrañaran los enfoques de enseñanza y aprendizaje que subyacen en las acciones deterministas en el uso didáctico de las tecnologías, y lo que es más importante aún, las consecuencias derivadas de dicha intervención en la práctica educativa, tanto para el profesorado como para el alumnado.

2. Reflexionaran sobre algunas ideas y creencias previas sobre cómo y qué trabajan, con o sin tecnologías, para detectar fortalezas y debilidades, miedos, incertidumbres y potencialidades. En otro lugar, nos hemos referido recientemente (Rayón y Rodríguez, 2006) a las dudas que asaltan al profesorado cuando comienza a experimentar una utilización de las TIC con voluntad transversal. Los interrogantes son numerosos: ¿habrá que eliminar contenidos?, si fuera así, ¿cuáles?, ¿qué tareas serían más pertinentes plantear a los alumnos? ¿podrían llegar a saber menos nuestros alumnos? ¿qué estrategias de trabajo habrá que poner en marcha y al servicio de qué metas educativas? ¿qué hacemos con el libro de texto? Como señalaba en el apartado anterior la formación del profesorado que ha dominado hasta el momento ha fragmentado e instrumentalizado en exceso el conocimiento didáctico en relación con la utilización de los medios tecnológicos, parece oportuno pues que trabajemos en otra dirección.

\subsection{En su formación.}

Acudiendo a Smyth (1991) compartimos que los docentes sólo podemos reclamar a los grupos ajenos a la escuela el poder que hemos perdido si nos enfrentamos a nuestros propios problemas con espíritu crítico, lo que no sólo tiene que ver con desarrollar acciones reflexivas en relación a nuestro trabajo, también con el desarrollo de la confianza en nuestra propia capacidad. 
Sin embargo, para generar confianza en los docentes y según este mismo autor "tiene menos que ver la transmisión de conocimientos que con el hecho de compartir mutuamente ideas, intuiciones y experiencias"(p. 278)

Esto, como hemos podido constatar en nuestro proceso de trabajo, no sucede cuando se realiza formación en TIC. Por el contario, los artefactos tecnológicos adquieren más protagonismo y sobrevaloración que como consecuencia de la función de vigilancia que ahora veremos más detenidamente - refuerza el aislamiento profesional, poniéndose de manifiesto que la organización, gestión de espacios y tiempo de formación responde a una serie de principios que legitiman el poder y vigilancia que agentes externos ejercen sobre el profesorado y la individualización como patrón relaciona de la cultura docente ${ }^{4}$

Se podría deducir, a partir de los datos, que esta realidad podría formar parte del expertísimo circundante, pero por su importancia para los objetivos de este artículo, hemos optado por darles un tratamiento más profundo en esta categoría, pues, de alguna forma, crea en los docentes una encrucijada que explica, en gran manera, lo expuesto hasta el momento.

Recurriendo a Foucault ${ }^{5}$ y a Bautista, A (1998) aplicado al campo de Internet, podemos distinguir dos técnicas de poder:

a) Las reguladoras de la vida social y de las poblaciones a través de un sistema legal. Entendemos, que la tecnología puede asumir esta función si se considera como soporte de la legislación que oriente sobre el papel de las TIC y de la utilización que debe hacer los usuarios y usuarias (docentes) con dichos medios. Esta función se está ejerciendo si analizamos diferentes disposiciones de ámbito nacional y/o autonómico.

- Nacional:

- Autonómico: Resolución de 24-07-2008, de la Dirección General de Política Educativa, por la que se aprueban las instrucciones para la planificación y elaboración del Plan Regional de Formación Permanente del Profesorado de Castilla - La Mancha para los cursos 2008-2009 y 2009-2010. (Hasta la fecha de la recogida de datos)

\footnotetext{
${ }^{4}$ Para CASTELL, M (1997) "el nuevo poder reside en los códigos de información y en las imágenes de representación en torno a los cuales las sociedades organizan sus instituciones y la gente construye sus vidas y decide su conducta. La sede de este poder es la sede de la mente". CASTELL, M (1997. La era de la información. El poder de la identidad. Vol. 2. Madrid: Alianza Editorial

${ }^{5}$ Gobernabilidad: "entiendo el conjunto constituido por las instituciones, los procedimientos, análisis y reflexiones, los cálculos y las tácticas que permiten ejercer esta forma tan específica, tan compleja, de poder; que tiene como meta principal la población, como forma primordial de saber; la economía política, como instrumento técnico esencial, los dispositivos de seguridad". M. Foucaul "La «gouvemementalité»"; curso del Collège de France, año 1977-1978. «Seguridad, territorio y población», $4^{\circ}$ lección, 1 de febrero de 1978, Aut-Aut, n 167-168, septiembre-diciembre de 1978, págs. 12-29.
} 
Es una forma de orientar el comportamiento de los docentes, una forma de gobernarlos

b) Las disciplinarias que son individualizantes y están centradas en los cuerpos (Foucault, 1979). Se fundamentan en el conocimiento generado en las diferentes áreas del saber correspondientes a objetivaciones que se hacen del sujeto: loco - cuerdo, sano - enfermo, experto - no experto. El conocimiento es poseído por el experto y lo aplicará cuando algún sujeto lo demande por tener dificultades (no experto). Este es el sentido disciplinar de las TIC, pues cada herramienta tecnológica que tenga un docente, va a ser punto de dependencia de los expertos correspondientes $y$, por consiguiente, va ser su punto de gobernabilidad en los momentos que tenga dificultad con alguno de ellos.

Esta organización y gestión de las TIC, favorece el control de la formación que llevan a cabo los docentes con los que hemos compartido esta investigación.

Cerrando este apartado, otro modelo de formación es posible, después de lo observado y trabajado con los docentes en formación, se identifican tres tipos de conocimientos necesarios para una integración de las TIC acorde con la sociedad de la información, que invierte el proceso de adquisición de dichos conocimientos.

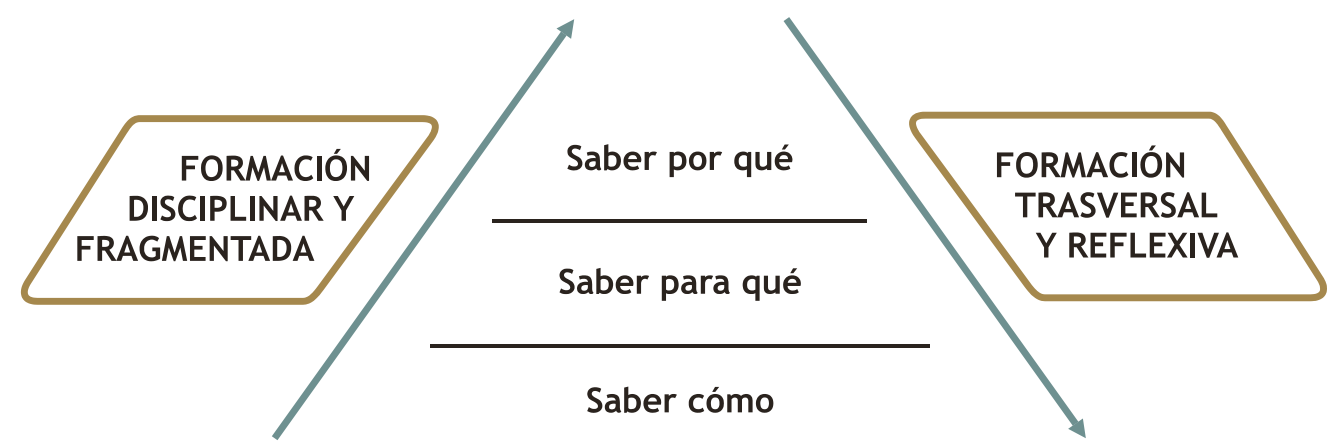

Necesidad de inversión del proceso

Se debe de tratar de que los docentes no empiecen centrando su atención únicamente en un saber cómo a nivel usuario de informática. Sabemos que es más sencillo comenzar por la base, y como hemos señalado esa es la tónica dominante en la permanente del profesorado, pero también hemos visto que a los docentes les será más difícil vislumbrar esos saberes transdisciplinares necesarios en la educación obligatoria hoy: seleccionar información, buscar datos relevantes, problematizar dichos datos para llegar a construir conocimiento (distinguir conocimiento de información), comunicar de un modo responsable y haciendo uso de los distintos códigos de representación que nos brindan las tecnologías, ... resultándoles también más difícil que puedan trascender prácticas instructivas y reproductoras.

En el terreno de la educación universitaria nos adentramos en este estudio en la manera en que los desarrollos de las nuevas tecnologías de la información afectan a la 
enseñanza del periodismo escrito y su inclusión en los medios de comunicación tanto dentro de la red como impresos. Dejamos de lado la faceta audiovisual para centrarnos en los medios escritos, que no impresos ya que los medios online tienen también esa virtud de la escritura.

Hemos estudiado, de esta forma, qué medios tienen de forma institucional las diversas universidades para desarrollar la labor comunicadora y docente. Pero, sobre todo, si esos medios están realizados desde la propia universidad o se ofrece la oportunidad al alumnado para que participe activamente en la creación y desarrollo de los mismos. Como quiera que el panorama es amplísimo, nos hemos centrado en estudiar algunos casos prácticos para realizar una investigación comparativa entre algunas universidades de las de mayor prestigio internacional y compararlas con una selección de algunas universidades españolas.

\section{CONCLUSIONES}

La lección aprendida con los docentes, como el lector- a ha tenido oportunidad de comprobar, se basaría en invertir la pirámide de modo que sea el extremo superior, donde están las razones y argumentos que justificarían la utilización didáctica de las TIC, las primeras cuestiones desde donde abordar la integración curricular de estos recursos. Para pasar posteriormente al nivel intermedio, el saber qué fines debe y pueden ser trabajados en función de los procesos y experiencias educativas que nos brindan estos recursos. Así, los docentes estarán en mejor disposición de traducir de un modo coherente y en esquemas prácticos aquello que hayan decidido es importante para su tarea docente en los otros dos niveles superiores. El cómo trabajar con las TIC estará apoyado y fundamentado en estos niveles, y no al revés.

No podemos terminar sin señalar lo que alguno de ustedes se estará preguntando, y que como tal expresamos en interrogante: ¿dónde situar la necesaria y mínima alfabetización instrumental para el conocimiento de las aplicaciones tecnológicas que los docentes deben dominar?

Sabemos, es verdad, que muchos docentes tienen <<miedo escénico>> en el momento de hacer uso en el aula de los recursos tecnológicos, por no tener una alfabetización que les permita manejar con cierta seguridad estos medios.

Sin embargo, nuestra experiencia docente y nuestra participación en la formación inicial nos ha demostrado que los profesores se alfabetizan en el manejo de los medios cuando sienten la necesidad de hacerlo, cuando las rutinas y destrezas que empleamos en el uso del ordenador, dvd, teléfono móvil... quedan subordinadas a propuestas de trabajo de más largo alcance, porque son significativas y relevantes para aprender otros conocimientos. Por ello, empezar por el primer nivel señalado no es óbice para poner al profesorado en situación de comenzar o perfeccionar destrezas técnicas de uso de las tecnologías y materiales multimedia a ellas asociados. Los entornos virtuales, como apoyo a la formación presencial, son una oportunidad para trabajar al unísono estos tres tipos de conocimiento. 


\section{BIBLIOGRAFÍA}

Accino, J. A. (1999). El silencio de los corderos: sobre las tecnologías de la información y la educación. Revista electrónica Heuresis, Vol.2, no 3

Bautista, A. (1998): Tecnología, mercado y gobernabilidad: un trinomio interactivo en la enseñanza a finales del segundo milenio. Revista Complutense de Educación, 9 (1), pp. 29-46.

Bautista, A. (2007). Alfabetización tecnológica multimodal e intercultural. Revista de Educación, 343. pp. $589-600$

Carbonell, J. (2001). La aventura de innovar. Madrid. Morata.

Foucault, M. (1979): Microfísica del Poder. Madrid. La Piqueta.

Fullan, M. (2002). Los nuevos significados del cambio en la educación. Barcelona. Octaedro.

Gimeno, J. (1996). La transición a la educación secundaria. Discontinuidades en las culturas escolares. Madrid, Morata.

Gimeno, J. (1999): Políticas y prácticas culturales en las escuelas: los abismos de la etapa postmoderna. Revista electrónica Heuresis, vol.2, nº1.

http://www2.uca.es/HEURESIS/heuresis99/v2n1.html

Gimeno, J. (2005). La educación que aún es posible. Madrid. Morata.

Martínez, J. (2002) Políticas del libro de texto escolar. Madrid. Morata.

Martinez, J. Et al. (2003). Ciudadanía, poder y educación. Barcelona. Graó.

Pérez, A. (1998): La cultura escolar en la sociedad neoliberal. Madrid. Morata.

Rayón, L. y Rodríguez, J. (2006). La necesaria $<<$ voz $>>$ del docente para la integración curricular de las TIC. OGE, no 4.

Smyth, J. (1991). "Una pedagogía Crítica de la práctica en el aula". Revista de Educación, no 294, $275-300$.

\section{Javier Rodríguez Torres}

Doctor en Pedagogía por la Universidad de Alcalá, Licenciado en Pedagogía y Diplomado en Magisterio por la Universidad Complutense de Madrid, Máster en Psicología Escolar por la misma Universidad. Más de veinte años en la Enseñanza no universitaria, ejerciendo como Profesor de Pedagogía Terapéutica, Profesor de Diversificación Curricular, Idioma (francés), Orientador de E. Infantil y Primaria. Desde 
el curso 2004 Profesor en la Universidad de Castilla - La Mancha, en el Departamento de Pedagogía, impartiendo Asignaturas de Teoría e Historia y DOE. En la actualidad, Secretario del Departamento. Profesor de distintos cursos de Postgrado de la UCLM en relación a la Atención temprana, Educación del Consumidor y del Máster de Secundaria. Premios nacionales, autonómicos y provinciales a distintos proyectos de investigación e innovación educativa. 